\title{
A STUDY ON MARKETING ACTIVITIES AND LISTENERS' ATTITUDES TOWARDS RADIO 94.3 MYFM: AN EMPIRICAL INVESTIGATION
}

\section{Dr. Mamta Brahmbhatt}

\begin{abstract}
This research basically focuses on Listeners' attitudes towards 94.3 MY FM. This survey focuses on radio listening and specifically the listening patterns and attitudes of MY FM listeners. Success in the ratings is determined by many factors. One of the most important is obviously a solid programming structure. Formats are the foundation to build the radio station on. They also show audience what to expect from the radio company. One should keep his/her promise every time that people tune in, and they'll grow into more loyal fans with every listening experience. This research explains about the choice of the listeners. What listeners want to listen, which type of music Listeners want to listen, the name of their favourite RJ, Listeners' behaviour towards the advertisements made by 94.3 MY FM. The likings towards the activities and events which 94.3 MY FM hosts etc. are also analysed.

Acknowledgement:

The author thank Tushar Rami and Akash Mistry for helping in collection of data and also thank respondents for taking part in survey.
\end{abstract}

\section{INTRODUCTION}

The impact of radio advertisements on people towards buying behaviour in retail stores and attempts to determine the role of radio advertising on dissemination of information on the sales promotions. The impact of radio advertisements on the store choice and buying preferences are analyzed based on empirical investigation.

With the changing mass communication scenario, Audience Research has occupied the centre stage. World over, almost all the big media organizations have been doing in-house audience research in one form or the other. Without 'Market Research' (in marketing) no media organization can afford to put their precious resource at stake without knowing the potential audience (consumers) and market for their media content. Besides, they are also subscribing 
to syndicated research done by the various media and market research organizations. The secret behind the success of private TV and Radio channels lies in their capability to feel the pulse of audience through continuous audience research and to design and modify the programme content including presentation accordingly.

The radio companies have to work hard to get the attention of audience towards them because in radio companies, cash generates from the advertisement of various companies which are being played by these radio companies. So most important thing for any radio company is to increase the listenership then and then only it will get more advertisers.

Listeners have very different behaviour towards the radio. Some listeners tune radio only to listen the songs. While, some listeners tune radio to get the updates from RJs, some people tune the radio to listen the advertisement. There are also some people who tune into radio just to win the prizes by answering the contest questions asked by RJs. Some people are also there who want to listen the songs only and for this they keep changing the stations again and again. The current research focuses on Listeners' attitudes towards 94.3 MY FM.

\section{OBJECTIVES OF RESEARCH}

- To know the awareness of MY FM in Ahmedabad.

- To know the behaviour of listeners' towards the MY FM.

- To know the satisfaction of listeners' with the vouchers or prizes given by MY FM.

- To know the loyalty of listeners towards MY FM.

\section{RESEARCH METHODOLOGY}

\section{Sample size determination}

A survey is being planned to determine what proportion of people in a certain region are satisfied with MYFM radio station. It is believed that the proportion cannot be greater than 0.20 A 95 percent confidence interval is desired width $d=0.05$.

Now using the formula given below:

$$
n=\frac{z^{2} p q}{d^{2}}
$$

Here $Z=1.96, p=0.20, q=0.80, d=0.05$,

$$
\boldsymbol{n}=\frac{(1.96)^{2}(0.20)(0.80)}{(0.05)^{2}}
$$

$\mathrm{n}=245.8624 \approx 246$ 


\section{Sample}

The population comprised listeners as defined in similar studied in literature. The target population of this study was composed of listeners of MYFM. A total of 65 questionnaires were distributed at four different places: total respondents surveyed are more than determined size which comes to 260 (65 respondents for each city).

\section{Methodology}

This is an analytical study based on the primary data collected through scientifically developed questionnaire. The questionnaire has been personally administered on sample size of 260 , chosen on a convenient basis from the four cities of Gujarat. Single Cross Sectional Descriptive Research Design has been used in the research. Secondary research was conducted and instruments used to measure listener's attitude Third, a questionnaire was constructed and piloted. Total 33 questions were included, which were divided into three parts, few are of Semantic differential scale type questions. For the analysis of data statistical methods are applied with the aid of SPSS (Statistical Package for Social Science) software, version 16.0 .

Table: 1 Characterstics of the sample

\begin{tabular}{|c|c|c|}
\hline & Category & Frequency \\
\hline \multirow{4}{*}{ AGE } & $15-20$ & 33 \\
\cline { 2 - 3 } & $20-25$ & 108 \\
\cline { 2 - 3 } & $25-35$ & 68 \\
\cline { 2 - 3 } & $35-55$ & 38 \\
\cline { 2 - 3 } & $55 \&$ above & 13 \\
\cline { 2 - 3 } Income & Total & $\mathbf{2 6 0}$ \\
\hline \multirow{4}{*}{} & Below 15000 & 46 \\
\cline { 2 - 3 } & $15000-30000$ & 100 \\
\cline { 2 - 3 } & $30000-45000$ & 56 \\
\cline { 2 - 3 } & $45000-60000$ & 31 \\
\cline { 2 - 3 } & above 60000 & 27 \\
\cline { 2 - 3 } & Total & $\mathbf{2 6 0}$ \\
\hline \multirow{4}{*}{ Occupation } & Employee & 29 \\
\cline { 2 - 3 } & Business & 50 \\
\cline { 2 - 3 } & house wife & 17 \\
\cline { 2 - 3 } & Student & 164 \\
\cline { 2 - 3 } & Total & $\mathbf{2 6 0}$ \\
\hline \multirow{4}{*}{ Gender } & Male & 199 \\
\cline { 2 - 3 } & Female & 61 \\
\cline { 2 - 3 } & Total & $\mathbf{2 6 0}$ \\
\hline
\end{tabular}

\section{DATA ANALYSIS AND INTERPRETATIONS}


Table: 2 T Test of gender and satisfaction towards 94.3 MY FM

\begin{tabular}{|l|l|l|l|l|}
\hline Independent Samples Test & \multicolumn{3}{|l|}{$\begin{array}{l}\text { t-test for } \\
\text { Equality of } \\
\text { Means }\end{array}$} & \multicolumn{2}{|l|}{ Result } & $\begin{array}{l}\text { Null } \\
\text { Hypothesis } \\
\text { Decision }\end{array}$ \\
\cline { 2 - 5 } & T & $\begin{array}{l}\text { Sig. } \\
(2- \\
\text { tailed) }\end{array}$ & & \\
\hline $\begin{array}{l}\text { Satisfaction level towards 94.3 MY FM (On air } \\
\text { contest) }\end{array}$ & 0.841 & 0.402 & $0.402>0.05$ & $\begin{array}{l}\text { Do not } \\
\text { reject }\end{array}$ \\
\hline $\begin{array}{l}\text { Satisfaction level towards 94.3 MY FM (RJ links) } \\
\text { Satisfaction level towards 94.3 MY FM (songs) }\end{array}$ & 2.018 & 0.46 & $0.46>0.05$ & $\begin{array}{l}\text { Do not } \\
\text { reject }\end{array}$ \\
\hline $\begin{array}{l}\text { Satisfaction level towards 94.3 MY FM (on ground } \\
\text { contest) }\end{array}$ & 2.622 & 0.10 & $0.10>0.05$ & $\begin{array}{l}\text { Do not } \\
\text { reject }\end{array}$ \\
\hline $\begin{array}{l}\text { Satisfaction level towards 94.3 MY FM (Meet and } \\
\text { Greet) }\end{array}$ & 0.934 & 0.353 & $0.353>0.05$ & $\begin{array}{l}\text { Do not } \\
\text { reject }\end{array}$ \\
\hline $\begin{array}{l}\text { Satisfaction level towards 94.3 MY FM (Unplugged } \\
\text { hours) }\end{array}$ & 0.762 & 0.448 & $0.448>0.05$ & $\begin{array}{l}\text { Do not } \\
\text { reject }\end{array}$ \\
\hline
\end{tabular}

There is no significant difference in satisfaction when classified by gender, so no need to design separate format based on gender.

Table: 3 T test of Gender and Sales promotion schemes of 94.3 MY FM.

\begin{tabular}{|c|c|c|c|c|}
\hline \multicolumn{5}{|l|}{ Independent Samples Test } \\
\hline & \multicolumn{2}{|c|}{$\begin{array}{l}\text { t-test for } \\
\text { Equality of } \\
\text { Means }\end{array}$} & \multirow[b]{2}{*}{ Result } & \multirow{2}{*}{$\begin{array}{l}\text { Null } \\
\text { Hypothesis } \\
\text { Decision }\end{array}$} \\
\hline & $\mathrm{T}$ & $\begin{array}{l}\text { Sig. } \\
(2- \\
\text { tailed) }\end{array}$ & & \\
\hline $\begin{array}{l}\text { Satisfaction level towards sales promotion schemes } \\
\text { given by } 94.3 \text { MY FM (Movie tickets) }\end{array}$ & 2.253 & 0.027 & $0.027<0.05$ & Reject \\
\hline $\begin{array}{l}\text { Satisfaction level towards sales promotion schemes } \\
\text { given by } 94.3 \text { MY FM (Salon vouchers) }\end{array}$ & 0.688 & 0.493 & $0.493>0.05$ & $\begin{array}{l}\text { Do not } \\
\text { reject }\end{array}$ \\
\hline $\begin{array}{l}\text { Satisfaction level towards sales promotion schemes } \\
\text { given by } 94.3 \text { MY FM (Selfie stick) }\end{array}$ & 0.740 & 0.461 & $0.0461<0.05$ & Reject \\
\hline $\begin{array}{l}\text { Satisfaction level towards sales promotion schemes } \\
\text { given by } 94.3 \text { MY FM (Fastrack watches) }\end{array}$ & 0.786 & 0.434 & $0.434>0.05$ & $\begin{array}{l}\text { Do not } \\
\text { reject }\end{array}$ \\
\hline $\begin{array}{l}\text { Satisfaction level towards sales promotion schemes } \\
\text { given by } 94.3 \text { MY FM (power bank) }\end{array}$ & 1.559 & 0.122 & $0.122>0.05$ & $\begin{array}{l}\text { Do not } \\
\text { reject }\end{array}$ \\
\hline $\begin{array}{l}\text { Satisfaction level towards sales promotion schemes } \\
\text { given by } 94.3 \text { MY FM (food vouchers) }\end{array}$ & 0.769 & 0.444 & $0.444>0.05$ & $\begin{array}{l}\text { Do not } \\
\text { reject }\end{array}$ \\
\hline $\begin{array}{l}\text { Satisfaction level towards sales promotion schemes } \\
\text { given by } 94.3 \text { MY FM (Recharge voucher) }\end{array}$ & 1.022 & 0.310 & $0.310<0.05$ & Reject \\
\hline
\end{tabular}


Towards Excellence: An Indexed, Refereed \& Peer Reviewed Journal of Higher Education / Dr. Mamta Brahmbhatt / Page 181-188

\begin{tabular}{|l|l|l|l|l|}
\hline $\begin{array}{l}\text { Satisfaction level towards sales promotion schemes } \\
\text { given by 94.3 MY FM (Petrol voucher) }\end{array}$ & 0.985 & 0.327 & $0.327>0.05$ & $\begin{array}{l}\text { Do not } \\
\text { reject }\end{array}$ \\
\hline $\begin{array}{l}\text { Satisfaction level towards sales promotion schemes } \\
\text { given by 94.3 MY FM (Beauty hamper) }\end{array}$ & 1.734 & 0.086 & $0.086>0.05$ & $\begin{array}{l}\text { Do not } \\
\text { reject }\end{array}$ \\
\hline $\begin{array}{l}\text { Satisfaction level towards sales promotion schemes } \\
\text { given by 94.3 MY FM (Gold coins) }\end{array}$ & 1.328 & 0.187 & $0.187>0.05$ & $\begin{array}{l}\text { Do not } \\
\text { reject }\end{array}$ \\
\hline
\end{tabular}

There is significant difference in satisfaction towards sales promotion strategies when classified by gender in statements number 1, 3 and 7, so different strategies need to be designed for these variables.

Table: 4 One way Anova (age and sales promotion activities)

\begin{tabular}{|c|c|c|c|c|}
\hline \multicolumn{5}{|l|}{ One Way ANOVA } \\
\hline Particulars & $\mathrm{F}$ & Sig. & Result & $\begin{array}{l}\text { Null } \\
\text { Hypothesis } \\
\text { Decision }\end{array}$ \\
\hline $\begin{array}{l}\text { Satisfaction level towards } \\
\text { sales promotion schemes } \\
\text { given by } 94.3 \text { MY FM (Movie } \\
\text { tickets) }\end{array}$ & 0.894978 & 0.470197 & $0.470197<0.05$ & Reject \\
\hline $\begin{array}{l}\text { Satisfaction level towards } \\
\text { sales promotion schemes } \\
\text { given by } 94.3 \text { MY FM (Salon } \\
\text { vouchers) }\end{array}$ & 1.48593 & 0.212584 & $0.212584<0.05$ & Reject \\
\hline $\begin{array}{l}\text { Satisfaction level towards } \\
\text { sales promotion schemes } \\
\text { given by } 94.3 \text { MY FM (Selfie } \\
\text { stick) }\end{array}$ & 0.491222 & 0.742164 & $0.742164<0.05$ & Reject \\
\hline $\begin{array}{l}\text { Satisfaction level towards } \\
\text { sales promotion schemes } \\
\text { given by } 94.3 \text { MY FM } \\
\text { (Fastrack watches) }\end{array}$ & 0.206734 & 0.934111 & $0.934111<0.05$ & Reject \\
\hline $\begin{array}{l}\text { Satisfaction level towards } \\
\text { sales promotion schemes } \\
\text { given by } 94.3 \text { MY FM (power } \\
\text { bank) }\end{array}$ & 0.746173 & 0.562949 & $0.562949<0.05$ & Reject \\
\hline $\begin{array}{l}\text { Satisfaction level towards } \\
\text { sales promotion schemes } \\
\text { given by } 94.3 \text { MY FM (food } \\
\text { vouchers) }\end{array}$ & 0.257998 & 0.904109 & $0.904109<0.05$ & Reject \\
\hline $\begin{array}{l}\text { Satisfaction level towards } \\
\text { sales promotion schemes } \\
\text { given by } 94.3 \text { MY FM } \\
\text { (Recharge voucher) }\end{array}$ & 1.40402 & 0.238571 & $0.238571<0.05$ & Reject \\
\hline $\begin{array}{l}\text { Satisfaction level towards } \\
\text { sales promotion schemes } \\
\text { given by } 94.3 \text { MY FM (Petrol } \\
\text { voucher) }\end{array}$ & 1.268077 & 0.288011 & $0.288011<0.05$ & Reject \\
\hline
\end{tabular}




\begin{tabular}{|l|l|l|l|l|}
$\begin{array}{l}\text { Satisfaction level towards } \\
\text { sales promotion schemes } \\
\text { given by 94.3 MY FM } \\
\text { (Beauty hamper) }\end{array}$ & 1.03582 & 0.392933 & $0.392933<0.05$ & Reject \\
\hline $\begin{array}{l}\text { Satisfaction level towards } \\
\text { sales promotion schemes } \\
\text { given by 94.3 MY FM (Gold } \\
\text { coins) }\end{array}$ & 0.57171 & 0.683799 & $0.683799<0.05$ & Reject \\
\hline
\end{tabular}

When sales promotion strategies are compared with various age groups, significant difference were found.

\section{MAJOR FINDINGS}

MY FM have more popularity compare to others. Which seems that MY FM always come with new concept for making awareness in listeners i.e MY FM have done book fair , Arachnanigarjana for SwachtaAbhiyan, Live RathYatra Show Arachanaka Challenge, PayalKa Top 10.

According to our research listeners are satisfied with Rj Links, On ground Activity, Prizes and vouchers . So Listeners don't want any change from MY FM.

MY FM branding as per respondents, Branding of MY FM by media partner of any event is having more awareness then other branding . MY FM doing branding by giving umbrella to tea- stall or any any needy people which is consider as CSR activity also. MY FM doing their branding by many ways .

MY FM having their own marketing strategy. They are doing nice tie-up as aprice voucher for contest, They are giving very exclusive price, Any expensive or exclusive Price can attracts to any one, like to college students or housewife, or working woman. From our research study we can say that listeners are satisfied with different Price Vouchers.

MY FM plays all kind of songs exception English, MY FM their own show PayalKa Top 10 in which RJ Payal plays all that songs as per listeners demand. As per research listeners like to listen mix song like old and new. So as MY FM playing all kind of song as per the show so listeners will always love to listen MY FM .

MY FM fans are youth, young generation age of 20-29. This group are like to listen MY FM, And also like their contest.

According to our research study $45 \%$ people like to listen MY FM . There is very tough fight between Red Fmand MY FM but MY FM having more market value then red fm. 
Although there is not such recommendations for 94.3 MY FM because 94.3 MY FM is the best place to work in its own way, I would want to suggest some things :

\section{MANAGERIAL IMPLICATIONS}

94.3 MY FM can improvise the coordination. The Researcher has noticed that the coordination lacks in 94.3 MY FM. Because of the lined up activities and events, sometimes the employees fail to coordinate well and then problem occurs. So, If this coordination part would improvise then the employees including the RJs would be able to work effectively.

The other thing which the researcher would want 94.3 MY FM to improvise is the Branding Part. If we look around the city, one would hardly find its branding hoardings. Where in, its competitors Red Fm and Radio Mirchi has done a good amount of Branding.

\section{CONCLUSION}

MY FM is more popular compare to others and MY FM always comes with new concept for making awareness in listeners i.e MY FM has done book fair, Arachnanigarjana for SwachtaAbhiyan, Live RathYatra Show Arachanaka Challenge, PayalKa Top 10 and the most popular award show "JIYO DIL SE". So, Listeners are satisfied with 94.3 MY FM deliverables but it should keep inventing something new every time to increase its listenership.

- $\quad$ http://www.exchange4media.com/radio/my-fm-ke-rangrezz-paint-\%22swachhbharat\%22_58265.html

- http://www.exchange4media.com/industrybriefing/94.3-my-fm-launched-rj-archanajani-in-its-morning-primetime-show-salaam-ahmedabad_56086.html

- http://www.afaqs.com/news/story/40148_My-FM-raises-ad-rates-by-25-per-cent

- http://www.business-standard.com/article/companies/my-fm-and-fever-fm-enter-intoa-strategic-sales-alliance-113040300412_1.html

- http://www.bestmediainfo.com/2013/05/my-fm-introduces-new-segment-thebhagwat/

- http://www.techsciresearch.com/2984

- http://www.broadcastandcablesat.co.in/indian-fm-radio-story-and-the-changingfuture.html 
- http://www.indiantelevision.com/television/tv-channels/music-and-youth/broadcategorisation-of-fm-radio-in-india-industry-has-seen-double-digit-growth-140424

- https://en.wikipedia.org/wiki/List_of_Indian-language_radio_stations

- http://www.marklives.com/2014/09/talking-radio-radio-listenership-behaviourshifting/\#.VavS4_mqqko

- http://allindiaradio.gov.in/Information/Pages/Audience\%20Research\%20Survey.aspx

- $\quad$ http://www.mvel.in/industry_overview.html

- $\quad$ http://www.radioiloveit.com/radio-programming-radio-formats/how-listenerexpectations-influence-ratings/

- http://www.researchgate.net/publication/254188702_Impact_of_radio_advertisements _on_buying_behaviour_of_urban_commuters

- http://www.ey.com/IN/en/Newsroom/News-releases/PR_Indias-FM-radio-segmentto-generate-INR14-bn-in-2012-13

\section{Dr. Mamta Brahmbhatt}

Associate Professor

B.K. School of Business Management

Gujarat University

Navrangpura, Ahmedabad-380009

mamtabrahmbhatt23@gmail.com 